\title{
Resonantly suppressed transmission and anomalously enhanced light absorption in ultrathin metal films
}

\author{
I. S. Spevak ${ }^{1}$, A. Yu. Nikitin ${ }^{1,2}$, E. V. Bezuglyi ${ }^{3}$, A. A. Levchenko ${ }^{4}$, and A. V. Kats 1 因 \\ ${ }^{1}$ A. Ya. Usikov Institute for Radiophysics and Electronics NASU, 61085 Kharkov, Ukraine \\ 2 Departamento de Física de la Materia Condensada-ICMA, Universidad de Zaragoza, E-50009 Zaragoza, Spain \\ ${ }^{3}$ B. I. Verkin Institute for Low Temperature Physics NASU, 61103 Kharkov, Ukraine \\ ${ }^{4}$ Department of Physics, University of Minnesota, Minneapolis, MN 55455, USA
}

(Dated: November 21, 2018)

\begin{abstract}
We study light diffraction in the periodically modulated ultrathin metal films both analytically and numerically. Without modulation these films are almost transparent. The periodicity results in the anomalous effects, such as suppression of the transmittance accompanied by a strong enhancement of the absorptivity and specular reflectivity, due to excitation of the surface plasmon polaritons. These phenomena are opposite to the widelyknown enhanced transparency of periodically modulated optically thick metal films. Our theoretical analysis can be a starting point for the experimental investigation of these intriguing phenomena.
\end{abstract}

PACS numbers: 42.25.-p

Ten years ago Ebbesen et al. [1] reported on a pioneering observation of the enhanced light transmission through subwavelength hole arrays. Now being classical, this experiment stimulated large number of investigations focused on the diffraction by the optically thick metal films which were periodically modulated by holes, slits, surface corrugations, etc. The physical origin of the enhanced light transmission is the interaction between the eigenmodes of an opaque metal film and the incoming and outgoing waves [2]. More specifically, periodic modulation leads to the transformation of incoming photons into surface plasmon polaritons (SPPs) localized at the film interfaces. The subsequent back transformation of the excited SPPs into outgoing photons gives rise to the observed anomalous effects in the light reflection and transmission.

In this Letter, we predict in some sense the opposite phenomenon. We consider ultrathin films with the thickness $\ell$ smaller or comparable to the skin depth, $\ell \lesssim \delta$, which is an opposite case as compared to the widely studied configurations. For such films, SPPs are separated into long-range (LR) and short-range (SR) modes [3, 4, 5] with the dispersion relations modified due to the modulation. The light transmission through the homogeneous ultrathin film is rather high. We show that a periodic modulation of the dielectric permittivity of thin films results in the paradoxical optical effect - highlytransparent films become nearly opaque and highly-reflective. The region of such anomalous behavior is blue-shifted with respect to the position of the local maximum in the transmittance. Another amazing feature of the predicted phenomenon is an extraordinary enhanced absorptivity (up to 50\%). The latter is pronounced only in a narrow vicinity of the maximum of the amplitude of the resonantly excited SPP wave. This resonance point is situated between the transmittance maximum and minimum (Fano profile), which are caused by constructive and destructive interference.

To clarify the most essential physics of the effect, we examine the simplest case of one-dimensional modulation with symmetric dielectric surrounding. In our analytical approach, we develop a resonance perturbation theory based on the small

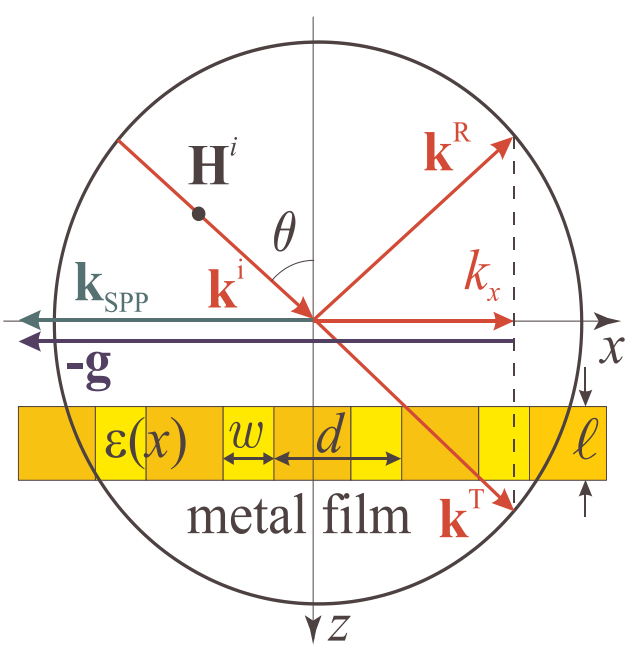

FIG. 1: (Color online) Geometry of the resonant diffraction problem. $\mathbf{k}^{i}, \mathbf{k}^{R}$, and $\mathbf{k}^{T}$ are the wave vectors of the incident, specularly reflected, and zero-order transmitted waves, respectively; $\mathbf{k}_{\mathrm{SPP}}$ is the wave vector of the excited SPP, $\mathbf{g}$ is the reciprocal grating vector, $d$ is the grating period, $w$ is the slit width.

parameter $\ell / \delta \ll 1$. Analogous analytical approach (with periodic modulation supposed to be a small parameter) has been successfully used before in studies of the resonant diffraction in periodically modulated metal half-spaces and thick films [6, 7, 8, 9]. Additional simplifications are provided by large negative values of the real part of the complex dielectric permittivity $\varepsilon=\varepsilon^{\prime}+i \varepsilon^{\prime \prime},\left|\varepsilon^{\prime}\right| \gg 1$, along with smallness of the absorption, $\varepsilon^{\prime \prime} \ll\left|\varepsilon^{\prime}\right|$. These conditions are typical for noble and some other metals in visible and near infrared spectral regions. Finally, our analytical results are supported by the numerical analysis.

Consider a plane monochromatic TM polarized wave, with magnetic field $\mathbf{H}^{i}(x, z)=\hat{\mathbf{y}} \exp \left(i \mathbf{k}^{i} \mathbf{r}\right)$, incident onto the periodically modulated metal film with the grating period $d$, 
as shown in Fig. 11 The wavevector of the incident wave $\mathbf{k}^{i}=k \sqrt{\varepsilon_{d}}(\sin \theta, 0, \cos \theta)$, where $k=\omega / c, \varepsilon_{d}$ is the dielectric permittivity of the surrounding medium, and $\theta$ denotes the angle of incidence. We represent the diffracted magnetic field in the half-spaces $z \leq 0, z \geq \ell$ in the form of the Fourier-Floquet expansion,

$$
H(x, z)=\sum_{n} \exp \left(i q_{n} x\right)\left\{\begin{array}{ll}
R_{n} e^{i f\left(q_{n}\right) z}, & z \leq 0 \\
T_{n} e^{-i f\left(q_{n}\right)(z-\ell)}, & z \geq \ell
\end{array},\right.
$$

where $f(q) \equiv \sqrt{\varepsilon_{d} k^{2}-q^{2}}$ with $\operatorname{Re}, \operatorname{Im} f(q) \geq 0, \quad q_{n}=$ $k \sqrt{\varepsilon_{d}} \sin \theta+n g, n=0, \pm 1, \ldots, g=2 \pi / d$ is the period of the reciprocal lattice, and the time dependence, $\exp (-i \omega t)$, is omitted. $R_{n}$ and $T_{n}$ are the reflection and transmission transformation coefficients (TCs), respectively. Inside the film, $0 \leq z \leq \ell$, the fields are

$$
\{H(x, z), \mathbf{E}(x, z)\}=\sum_{n}\left\{H_{n}(z), \mathbf{E}_{n}(z)\right\} \exp \left(i q_{n} x\right) .
$$

Excluding the normal component of the electric field by means of the Maxwell equations, we get the set of the firstorder linear ordinary differential equations for the functions $H_{n}(z), E_{x n}(z)$. The solution of this set couples the values of the internal fields on the interfaces. By matching the fields at the interfaces, we obtain an infinite set of linear algebraic equations for $R_{n}$ and $T_{n}$.

Before proceeding with the analytical treatment, we present an example of numerical calculations of the resonant spectral properties of a periodically modulated ultrathin film, Fig. 2. The calculations have been performed by the rigorous coupled wave analysis (RCWA) with the improvement suggested in [10, 11]. We assume an opaque filling of the slits which restricts the resonances to the SPP modes only and prevents other resonances (e.g., slit modes). Note that the opaque material of the filling does not qualitatively influence the results. Therefore, it is sufficient to chose a high-contrast but opaque filling resulting in strong resonances even for small slit widths.

Figure 2 shows that the transmittance $\left|T_{0}\right|^{2}$, absorptivity $A$, and reflectance $\left|R_{0}\right|^{2}$ possess strong resonant behavior for various slit widths $w$ in the spectral region typical for the experiments. Depending on the slit width, the transmittance in the resonance region is suppressed up to $10 \%$ for $\ell=10 \mathrm{~nm}$, whereas it is of order of $85 \%$ away from the resonance. In contrast, the reflectance and absorption are greatly enhanced up to $50 \%$. It turns out that all shown peculiarities are related to the resonant excitation of SR SPP in a certain diffraction order. Namely, the longest-wavelength extremes of $\left|R_{0}\right|^{2},\left|T_{0}\right|^{2}$ and $A$ correspond to the -1 st SR SPP resonance shown in Fig. 2 a, while the short-wavelength extremes are due to the +1 st and -2 nd resonances.

In order to explain quantitatively the resonant peculiarities of the spectra, we consider analytically the solution in the vicinity of a single SR resonance. To this end, we solve the set of equations for the field amplitudes in the resonant approximation [6, 7, 8, 9] using the small parameter $\ell / \delta=k \ell \sqrt{|\varepsilon|} \ll$ 1. The resonances correspond to the excitation of both SR
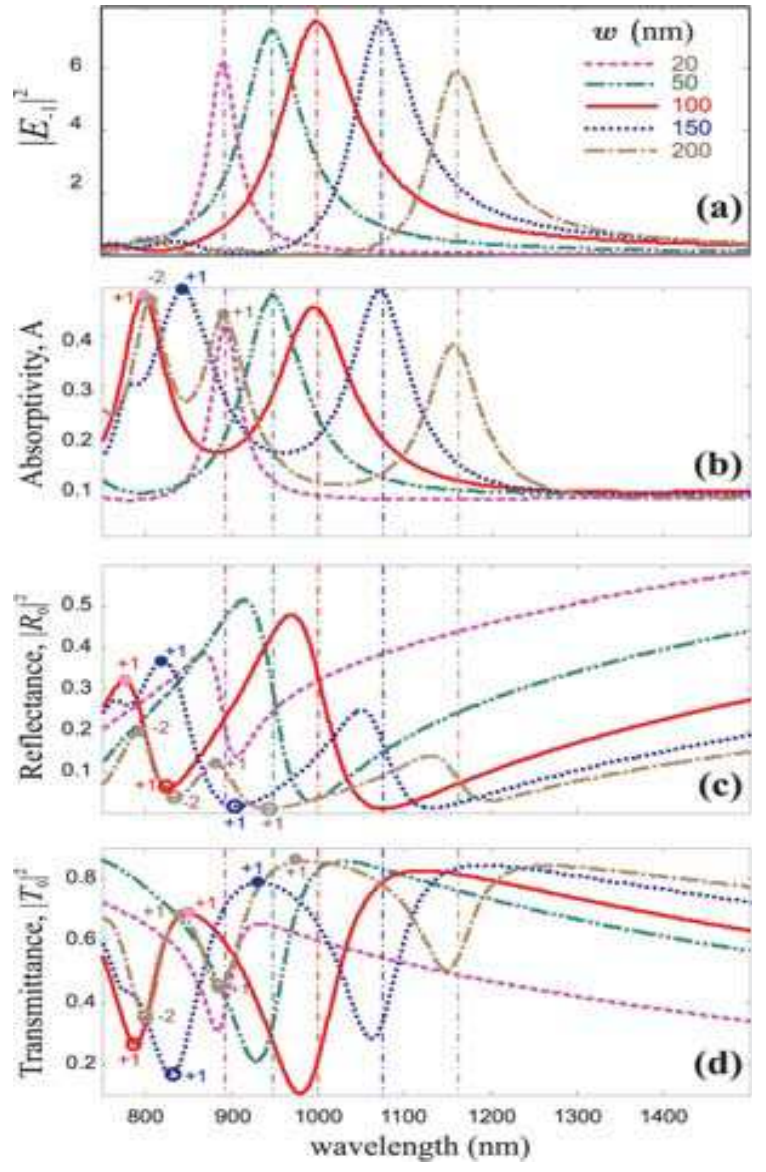

FIG. 2: (Color online) Wavelength dependence of the -1 st order intensity at the film boundary (a), total absorptivity (b), zero diffraction order reflectance (c), and transmittance (d) (numerical calculation). The parameters are: $\varepsilon_{d}=1, \varepsilon_{\text {lamel }}=\varepsilon_{\text {gold }}, \varepsilon_{\text {slit }}=0.2 \varepsilon_{\text {gold }}$ (the wavelength-dependent gold permittivity is taken from Ref. [12]), the incident angle $\theta=45^{\circ}$, the grating period $d=400 \mathrm{~nm}$, the film thickness $\ell=10 \mathrm{~nm}$, the slit width $w$ is shown in the legend in (a). Vertical dash-dot lines indicate the position of the SR resonance in $r=-1$ diffraction order; open (filled) circles indicate minima (maxima) of $\left|R_{0}\right|$ and $\left|T_{0}\right|$ for the $r=1$ and $r=-2$ resonances. The longest-wavelength extremes (not marked) correspond to the $r=-1$ resonance.

and LR SPP modes. However, as it follows from the analytical considerations and is confirmed by the computations, just the excitation of SR SPP strongly affects energy fluxes of the outgoing waves. Within the second order in $k \ell$, we obtain the resonant TC in the vicinity of a single SR resonance in $r$ th diffraction order,

$$
R_{r}=-\frac{u_{0}}{u_{0}+2 i} \frac{\mathbb{E}_{r 0}}{\mathbb{E}_{00}} \frac{1}{F_{r} / F_{S R}-1}, \quad T_{r} \simeq-R_{r}
$$

where

$$
\begin{aligned}
F_{m} & =f\left(q_{m}\right) /\left(k \sqrt{\varepsilon_{d}}\right), \quad u_{m}=k \ell \mathbb{E}_{m m} F_{m}, \quad F_{S R}=2 / i k \ell Z_{r}, \\
Z_{r} & =\mathbb{E}_{r r}+i k \ell X_{r}, \quad X_{r}=\sum_{N \neq r} \frac{F_{N} \mathbb{E}_{r N} \mathbb{E}_{N r}}{2-i u_{N}} .
\end{aligned}
$$


Here $\widehat{\mathbb{E}}=\hat{\zeta}^{-1}$, where the elements of the Töplitz matrix $\hat{\zeta}$ are formed by the Fourier coefficients of $1 / \varepsilon(x)$ expansion, $\zeta_{m n}=(1 / \varepsilon)_{m-n}$, Refs. [10, 11].

The resonant TC is proportional to the modulation harmonic which couples the incident wave with SPP through a one-step scattering. Other scattering processes contribute to the denominator of $R_{r}$. The pole of $R_{r}$ corresponds to the SR SPP dispersion relation $q_{S R}^{2} \simeq \varepsilon_{d} k^{2}\left[1+4 \varepsilon_{d}\left(k \ell Z_{r}\right)^{2}\right]$ modified by a periodic modulation. This modification is governed by the real part of $Z_{r}, Z_{r}^{\prime} \simeq \varepsilon_{0}^{\prime}-k \ell X_{r}^{\prime \prime}$, which is basically determined by the first term. This explains naturally the red-shift of the resonant peculiarities with increasing slit width $w$ (see Fig. 2. Indeed, when $w$ increases, $\left|\varepsilon_{0}^{\prime}\right|$ decreases and forces the red-shift of the SPP dispersion relation, as demonstrated in Fig. 3.

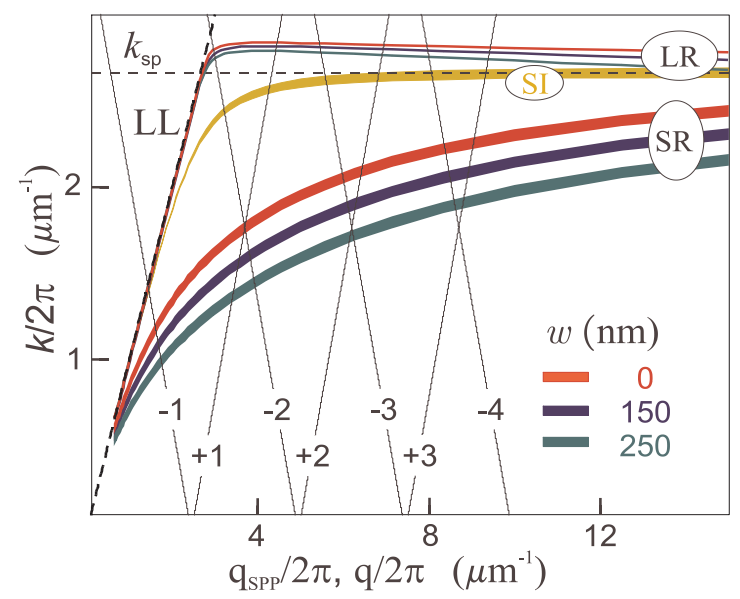

FIG. 3: (Color online) Dispersion curves for SPPs at the air/gold single interface (SI), and for the SR and LR SPPs for a homogeneous film with mean permittivity $\varepsilon_{0}$ for different slit widths and $\ell=10 \mathrm{~nm}$. The thickness of these curves reflects the SPP linewidth. The light line $(L L)$ is shown in black dash. The dashed horizontal line indicates the surface plasmon frequency, $k_{s p}=\omega_{s p} / c, \varepsilon^{\prime}\left(\omega_{s p}\right)=-1$. Numbered inclined lines are $q=\left|q_{n}\right|=|k \sin \theta+n g|$ with $n= \pm 1, \ldots$ for $\theta=45^{\circ}$. Intersection of $n$th line with the dispersion curve corresponds to the resonance excitation of the SPP in $n$th diffraction order.

The dissipation and SPP linewidth are governed by the imaginary part of $Z_{r}, Z_{r}^{\prime \prime} \simeq \varepsilon_{0}^{\prime \prime}+k \ell X_{r}^{\prime}$. Here the second term exceeds the first one even for a moderate modulation. Emphasize that the second term is mainly determined by the summation over outgoing waves [in Eq. [5]] responsible for SPP radiative losses (leakage).

We show in Fig. 4h an example of the resonant field amplitude calculated from Eqs. (3)-(5) for $r=-1$. Due to the excitation of SR SPP, $R_{r}$ approaches maximum in the vicinity of the SPP pole at the point $F_{r}=F_{S R}$ [see Figs. 2a and [4]]. In view of $\varepsilon^{\prime \prime} \ll\left|\varepsilon^{\prime}\right|$, the pole is close to the imaginary axis in the complex $F_{r}$ plane. The condition $\operatorname{Im}\left[F_{r}(\lambda, \theta)\right]=$ $\operatorname{Im}\left[F_{S R}(\lambda, \theta)\right]$ yields the local maximum of $\left|R_{r}\right|$ in $(\lambda, \theta)$
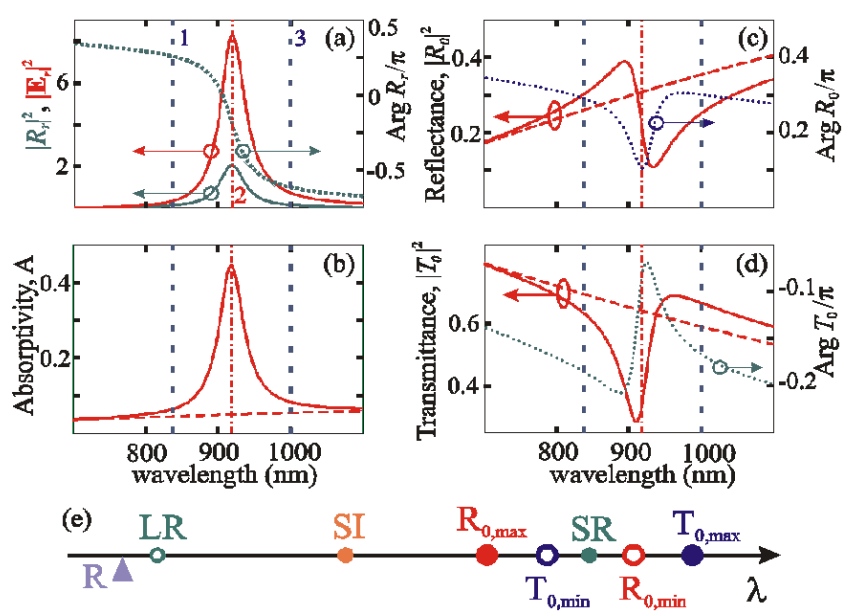

FIG. 4: (Color online) Results of analytical calculations for $r=-1$ resonance: wavelength dependence of the resonant wave magnitude and phase (a), total absorptivity (b), zero-order reflectance (c), and transmittance (d) for the slit width $w=100 \mathrm{~nm}$; other parameters are the same as in Fig. 2 Vertical lines 1-3 indicate the wavelengths of: SR SPP for slitless gold film (1) and the SR SPP resonance for modulated film calculated analytically (2) and numerically (3), cf. Fig. 2 Dash lines in (b)-(d) show quantities for a homogeneous film with the mean permittivity value. The typical ordering of the Rayleigh point, R, the long-range (LR), single-interface (SI), short-range (SR) $\mathrm{SPP}$ resonances, and the extreme points for the zero-order reflectance and transmittance are shown in (e).

plane. Along this resonance line we have

$$
\left|R_{r}\right|_{\max }^{2} \simeq \frac{\left|u_{0}\right|^{2}}{\left|u_{0}\right|^{2}+4}\left|\frac{\mathbb{E}_{r 0}}{\mathbb{E}_{00}}\right|^{2}\left(\frac{Z_{r}^{\prime}}{Z_{r}^{\prime \prime}}\right)^{2} .
$$

To study the far field, the knowledge of the nonresonantorder TCs is necessary. In general, not only the zero-order but also other diffracted orders contribute to the far-field energy flux. Within our analytical approach, they are given by

$$
R_{N}=\bar{R}_{N}+\delta R_{N}, \quad T_{N}=\bar{T}_{N}-\delta R_{N}, \quad N \neq r
$$

where $\bar{R}_{N}, \bar{T}_{N}$ are contributions from the nonresonant scattering processes,

$$
\bar{R}_{N}=\frac{k \ell F_{0} \mathbb{E}_{N 0}}{u_{N}+2 i}, \quad \bar{T}_{N}=\delta_{N, 0}-\bar{R}_{N},
$$

and the term $\delta R_{N}$ is the resonant contribution coming from the SR SPP back-scattering into the nonresonant diffraction orders,

$$
\delta R_{N}=-k \ell \frac{\left(F_{r}-i k \ell / 2\right) \mathbb{E}_{N r}}{u_{N}+2 i} R_{r}
$$

For zero-order TCs, $T_{0}$ and $R_{0}$, the nonresonant terms coincide with corresponding Fresnel coefficients $\bar{T}_{0}$ and $\bar{R}_{0}$ for the film with mean value of the dielectric permittivity, $\mathbb{E}_{00} \simeq \varepsilon_{0}$. Spectral dependences of $\left|\bar{T}_{0}\right|^{2}$ and $\left|\bar{R}_{0}\right|^{2}$ are shown by dash 
lines in Fig. 4 4 , $4 \mathrm{~d}$. Thus, $T_{0}$ and $R_{0}$ result from the interference between the resonant and direct (nonresonant) channels. This interference leads to neighboring minima and maxima in both $\left|R_{0}\right|$ and $\left|T_{0}\right|$ (Fano profile), see panels (c) and (d) in Figs. 4, 2 According to Eqs. (7)-(9), the maximum of $\left|T_{0}\right|^{2}$ lies to the right from the above indicated resonant point, whereas the minimum lies to the left. The maximum of $\left|T_{0}\right|^{2}$ is accompanied by the complementary minimum of $\left|R_{0}\right|^{2}$ and vice versa. Positions of these complementary extrema are slightly different, as shown in Fig. 4 . The resonance also results in the absorption maximum (in the close vicinity of $\left|R_{r}\right|^{2}$ maximum), cf. Figs. 4, 2] Noteworthy, even for small active losses, $\varepsilon^{\prime \prime} \ll\left|\varepsilon^{\prime}\right|$, the absorption, being small for ultrathin unmodulated films, increases substantially (up to 50\%) for an appropriate modulation.

Consider now in detail the case of $r=-1$ resonance. It is well separated from other resonances at oblique incidence. Moreover, only zero-order diffracted waves are outgoing and contribute to the far field. This leads to reduction of the radiative losses and resonant linewidth as compared with other resonances. One can see from Eq. (6) and Fig. 2a that the spectral maximum of the resonance-order intensity depends nonmonotonously on the modulation (e.g., on the slit width). The intensity is maximal at $\left|\mathbb{E}_{r 0}\right|=\mathscr{E}$, when the dissipation losses are equal to the radiative ones, i.e.,

$$
\mathscr{E}^{2}=\frac{\left|u_{0}\right|^{2}+4}{2\left|u_{0}\right|} \mathbb{E}_{00}^{\prime \prime}\left|\mathbb{E}_{00}\right|, \quad r=-1
$$

Substitution of $\mathscr{E}$ into Eq. (6) yields $\left|R_{r}\right|_{\max }^{2} \rightarrow\left|R_{r}\right|_{\text {extreme }}^{2} \simeq$ $\left|u_{0} \mathbb{E}_{00}\right| /\left(8 \mathbb{E}_{00}^{\prime \prime}\right) \gg 1$. For the modulation level given by Eq. [10), we obtain $T_{0}=R_{0}=1 / 2$, and the absorptivity approaches the maximal value 0.5 in the resonance. For slit structure with $\varepsilon_{d}=1, \varepsilon_{\text {lamel }}=\varepsilon_{\text {gold }}, \varepsilon_{\text {slit }}=0.2 \varepsilon_{\text {gold }}$, and $\theta=$ $45^{\circ}$, Eq. (10) gives the optimal value $w / d \simeq 1 / 4$ which well agrees with the numerical simulations, cf. Fig. 2 .

The minimum value of the transmittance also depends nonmonotonously on the slit width. This minimum can be very low (specifically, for transparent slits it may fall to few percents). The minimal value of the zero-order transmittance is determined by the sum of the dissipative and radiative losses in nonzero-order diffraction channels. For $r=-1$ resonance, the nonzero-order radiative losses are absent, and the transmittance falls up to $\left|T_{0}\right|_{\min } \propto \varepsilon_{0}^{\prime \prime} /\left|\varepsilon_{r}\right| \ll 1$.

The predicted effect of the transmittance suppression is illustrated in Fig. 5. Here we show the instant distribution of the magnetic field $\operatorname{Re}[H(x, z)]$ for two values of the wavelength related to the minimum and maximum of the transmittance, cf. Figs. 2k and 2 $\mathrm{d}$. The transmittance minimum is accompanied by the reflectance maximum. As a result, the high-contrast interference pattern appears, see Fig. 5a. On the other hand, the transmittance maximum is accompanied by the reflectance minimum resulting in almost complete disappearance of the interference, Fig. 5 b.

In conclusion, we have predicted the paradoxical resonant properties of ultrathin periodically modulated metal films. In
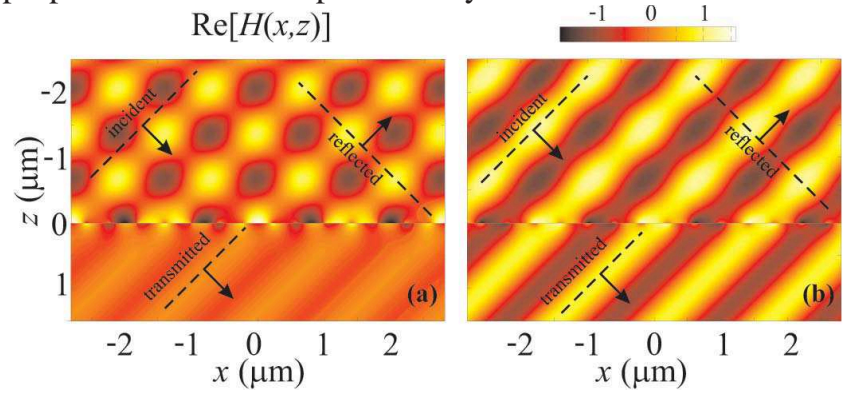

FIG. 5: (Color online) Spatial distribution of the magnetic field for $w=100 \mathrm{~nm}, \lambda=980 \mathrm{~nm}$ in (a), and $\lambda=1122 \mathrm{~nm}$ in (b). These wavelengths correspond to the transmittance minimum and maximum, respectively, for -1 st order resonance. Other parameters are the same as in Fig. 2

contrast to widely discussed optically thick films, where the modulation can result in extraordinary light transmission, the periodical modulation of ultrathin films can provide almost total suppression of the transmission. This effect is due to the resonance excitation of the short-range SPPs, which results also in up to 50 percents absorptivity and high reflectivity of the films.

The work was partially supported by STCU grant No 3979.

* Electronic address: ak_04@ rambler.ru

[1] T. W. Ebbesen et al., Nature 391, 667 (1998).

[2] L. Martín-Moreno et al., Phys. Rev. Lett. 86, 1114 (2001).

[3] H. Raether, Surface plasmons, (Springer-Verlag, New York, 1988).

[4] E. N. Economou, Phys. Rev., 182, 539 (1969).

[5] Z. Chen, I. R. Hooper, and J. R. Sambles, Phys. Rev. B 77, 161405(R) (2008).

[6] A. V. Kats, V. V. Maslov, Sov. Phys. JETP 35, N 2, 264 (1972).

[7] A. V. Kats, P. D. Pavitskii, I. S. Spevak, Sov. Phys. JETP 78, N 1, 42 (1994).

[8] A. V. Kats, N. A. Balakhonova, I. S. Spevak, Phys. Rev. B 76, 075407 (2007).

[9] A. V. Kats, M. L. Nesterov, A. Yu. Nikitin, Phys. Rev. B 76, 045413 (2007); ibid, 72, 193405 (2005).

[10] P. Lalanne and G. M. Morris, J. Opt. Soc. Am. A 13, 779784 (1996).

[11] L. Li, J. Opt. Soc. Am. A 13, N 9, 1870 (1996).

[12] S. G. Rodrigo, F. J. Garcia-Vidal, L. Martin-Moreno, Phys. Rev B 77, 075401 (2008). 